\title{
Explicit Exact Formulas for the 3-D Tetrahedron Inertia Tensor in Terms of its Vertex Coordinates
}

\author{
F. Tonon \\ Department of Geology and Geophysics, University of Utah \\ 135 South 1460 East, Room 719, Salt Lake City, UT 84112, USA
}

\begin{abstract}
The inertia tensor of a tetrahedron is composed of its moments of inertia. This study presents explicit exact formulas for the moments of inertia of a 3-D tetrahedron as simple polynomials of its vertex coordinates.
\end{abstract}

Keywords: Inertia tensor, inertia moments, tetrahedron

\section{INTRODUCTION}

The knowledge of the inertia tensor of a solid is of fundamental importance in many branches of applied mathematics, computer science and mechanics. For example, in mechanics the motion of a rigid body is controlled by the inertia tensor of a body through Euler's equations [1].

Besides their speculative interest as the simplest Platonic solids [2], tetrahedra are important in engineering: (e. g.) in rock mechanics the vast majority of unstable blocks are found to be tetrahedra [3, 4]. Moreover, tetrahedra properties are of interest in geometric modeling because any solid can be approximated as a polyhedron and an $n$-vertex polyhedron can be triangulated with $O\left(n^{2}\right)$ tetrahedra (if the polyhedron is convex, it can be triangulated with at most $2 n-7$ tetrahedra) [5]. If the tetrahedron inertia tensor can be easily calculated with respect to the same reference system, then the inertia tensor for the entire solid can be obtained as the sum of those inertia tensors. The solid's inertia tensor with respect to any other reference system can be calculated by rotating the tensor and using Steiner's theorem.

Although formulas have been given for the integration of polynomials over a tetrahedron [6-9] and references therein, in the literature no explicit expression has been given for the inertia tensor in terms of the vertex coordinates. This study provides these expressions with respect to a reference system of arbitrary origin and gives a numerical example for validation.

Formulation: Let $A_{\mathrm{i}}=\left(\tilde{x}_{i}, \tilde{y}_{i}, \tilde{z}_{i}\right) \quad i=1, \ldots, 4$ be the tetrahedron vertex coordinates with respect to a generic coordinate system $O, \tilde{x}, \tilde{y}, \tilde{z}$ (Fig. 1) and let $Q=\left(\tilde{x}_{Q}, \tilde{y}_{Q}, \tilde{z}_{Q}\right)$ be a point of the Euclidean 3-D space. Now, consider a new coordinate system whose axes $x$, $y, z$ are parallel to $\tilde{x}, \tilde{y}, \tilde{z}$, respectively and whose origin is $Q$; in the new system, the coordinates of the vertices are $A_{\mathrm{i}}=\left(x_{\mathrm{i}}, y_{\mathrm{i}}, z_{\mathrm{i}}\right), i=1, \ldots, 4$, where:

$\left\{\begin{array}{l}x_{i}=\tilde{x}_{i}-\tilde{x}_{Q} \\ y_{i}=\tilde{y}_{i}-\tilde{y}_{Q} \\ z_{i}=\tilde{z}_{i}-\tilde{z}_{Q}\end{array}\right.$

The inertia tensor $\mathbf{E}_{\mathrm{Q}}$ of a body $D$ with respect to the three axes $x, y, z$ centered at $Q$ is defined as [10]:

$\mathbf{E}_{Q}=\left(\begin{array}{ccc}a & -b^{\prime} & -c^{\prime} \\ -b^{\prime} & b & -a^{\prime} \\ -c^{\prime} & -a^{\prime} & c\end{array}\right)$

where, indicating with $\mu$ the density of the medium:

$$
\begin{aligned}
& a=\int_{D} \mu\left(y^{2}+z^{2}\right) d D ; b=\int_{D} \mu\left(x^{2}+z^{2}\right) d D ; \\
& c=\int_{D} \mu\left(x^{2}+y^{2}\right) d D \\
& a^{\prime}=\int_{D} \mu y z d D ; b^{\prime}=\int_{D} \mu x z d D ; c^{\prime}=\int_{D} \mu x y d D
\end{aligned}
$$

Quantities $a, b, c$ are the moments of inertia with respect to axes $x, y, z$, respectively and $a^{\prime}, b^{\prime}, c^{\prime}$ are the products of inertia. The principal directions are the eigenvectors of $\mathbf{E}_{\mathrm{Q}}$ and the principal moments of inertia are the eigenvalues of $\mathbf{E}_{\mathrm{Q}}$. If $x$ is a principal direction, 


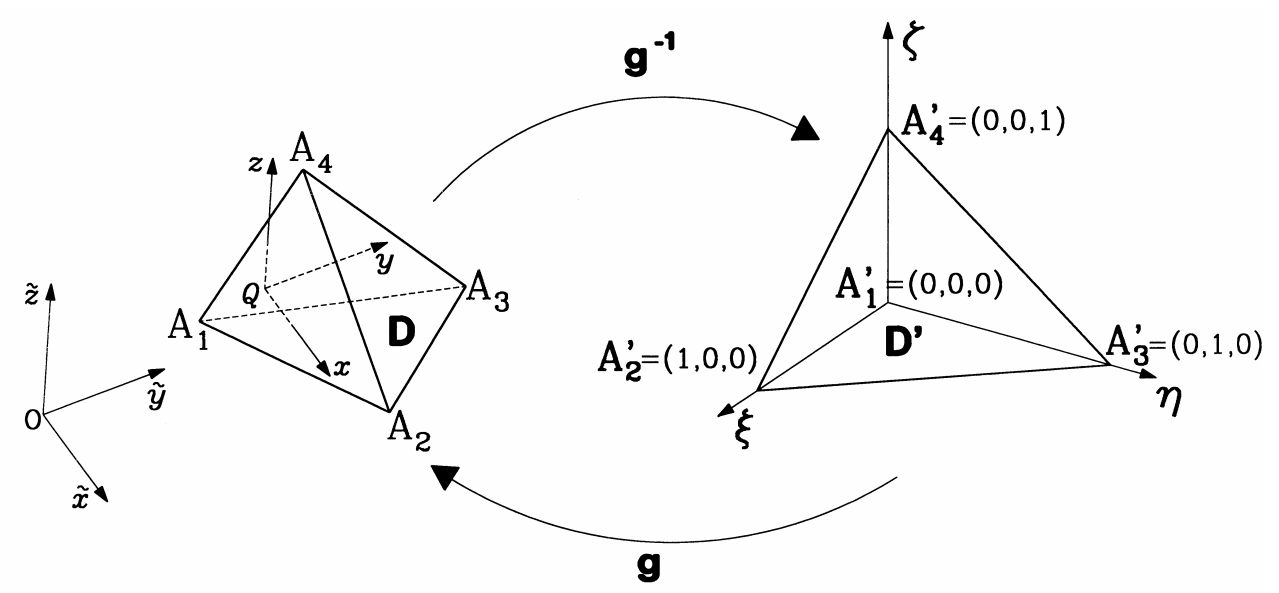

Fig. 1: Transformation $\mathrm{g}$ and $\mathrm{g}^{-1}$

then $b^{\prime}$ and $c^{\prime}$ are zero and so on. In order to calculate more easily the integrals in (3a) and (3b), let us change coordinate system by means of the following (affine) transformation $\mathbf{g}$ (Fig. 1):

$$
\left\{\begin{array}{l}
x=x_{1}+\left(x_{2}-x_{1}\right) \cdot \xi+\left(x_{3}-x_{1}\right) \cdot \eta+\left(x_{4}-x_{1}\right) \cdot \zeta \\
y=y_{1}+\left(y_{2}-y_{1}\right) \cdot \xi+\left(y_{3}-y_{1}\right) \cdot \eta+\left(y_{4}-y_{1}\right) \cdot \zeta \\
z=z_{1}+\left(z_{2}-z_{1}\right) \cdot \xi+\left(z_{3}-z_{1}\right) \cdot \eta+\left(z_{4}-z_{1}\right) \cdot \zeta
\end{array}\right.
$$

The determinant of the Jacobian is :

$$
\operatorname{DET}(J)=\left|\begin{array}{lll}
\frac{\partial x}{\partial \xi} & \frac{\partial x}{\partial \eta} & \frac{\partial x}{\partial \zeta} \\
\frac{\partial y}{\partial \xi} & \frac{\partial y}{\partial \eta} & \frac{\partial y}{\partial \zeta} \\
\frac{\partial z}{\partial \xi} & \frac{\partial z}{\partial \eta} & \frac{\partial z}{\partial \zeta}
\end{array}\right|=\left|\begin{array}{ccc}
x_{2}-x_{1} & x_{3}-x_{1} & x_{4}-x_{1} \\
y_{2}-y_{1} & y_{3}-y_{1} & y_{4}-y_{1} \\
z_{2}-z_{1} & z_{3}-z_{1} & z_{4}-z_{1}
\end{array}\right|=6 \cdot \text { Vol = constant }
$$

where, $\mathrm{Vol}$ is the volume of $D$.

Transformation $\mathbf{g}^{-1}$ normalizes tetrahedron $D$ into a tetrahedron $D^{\prime}$, (Fig. 1). Let now $f(x, y, z)$ be a generic function, continuous on $D$; since $\mathbf{g}$ is regular and $D$ is bounded by regular surfaces (planes), one can write:

$$
\int_{D} f(x, y, z) d D=\int_{D^{\prime}} f[x(\xi, \eta, \zeta), y(\xi, \eta, \zeta), z(\xi, \eta, \zeta)] \cdot|D E T(J)| d D^{\prime}
$$

Since $D^{\prime}$ is normal with respect to the $(\xi, \eta)$-plane and the projection of $D^{\prime}$ on plane $(\xi, \eta)$ is normal with respect to the $\xi$-axis, one has [11]:

$$
\int_{D} f(x, y, z) d D=|D E T(J)| \cdot \int_{0}^{1} d \xi \int_{0}^{1-\xi} d \eta \int_{0}^{1-\xi-\eta} f[x(\xi, \eta, \zeta), y(\xi, \eta, \zeta), z(\xi, \eta, \zeta)] d \zeta
$$

For example, if $D$ is homogeneous: 


$$
a=\mu \cdot \int_{D}\left(y^{2}+z^{2}\right) d D=\mu \cdot|D E T(J)| \cdot \int_{0}^{1} d \xi \int_{0}^{1-\xi} d \eta \int_{0}^{1-\xi-\eta}\left[y^{2}(\xi, \eta, \zeta)+z^{2}(\xi, \eta, \zeta)\right] d \zeta
$$

Integral (8) was solved and its solution checked using Mathematica:

$$
\begin{aligned}
& a=\mu \cdot|\operatorname{DET}(\mathrm{J})| \cdot\left(\mathrm{y}_{1}{ }^{2}+\mathrm{y}_{1} \mathrm{y}_{2}+\mathrm{y}_{2}{ }^{2}+\mathrm{y}_{1} \mathrm{y}_{3}+\mathrm{y}_{2} \mathrm{y}_{3}+\right. \\
& +\mathrm{y}_{3}{ }^{2}+\mathrm{y}_{1} \mathrm{y}_{4}+\mathrm{y}_{2} \mathrm{y}_{4}+\mathrm{y}_{3} \mathrm{y}_{4}+\mathrm{y}_{4}{ }^{2}+\mathrm{z}_{1}{ }^{2}+\mathrm{z}_{1} \mathrm{z}_{2}+ \\
& \left.+\mathrm{z}_{2}{ }^{2}+\mathrm{z}_{1} \mathrm{z}_{3}+\mathrm{z}_{2} \mathrm{z}_{3}+\mathrm{z}_{3}{ }^{2}+\mathrm{z}_{1} \mathrm{z}_{4}+\mathrm{z}_{2} \mathrm{z}_{4}+\mathrm{z}_{3} \mathrm{z}_{4}+\mathrm{z}_{4}{ }^{2}\right) / 60
\end{aligned}
$$

By proceeding in the same way for the other integrals one obtains:

$$
\begin{aligned}
& \mathrm{b}=\mu \cdot|\operatorname{DET}(\mathrm{J})| \cdot\left(\mathrm{x}_{1}^{2}+\mathrm{x}_{1} \mathrm{x}_{2}+\mathrm{x}_{2}^{2}+\mathrm{x}_{1} \mathrm{x}_{3}+\mathrm{x}_{2} \mathrm{x}_{3}+\mathrm{x}_{3}^{2}+\right. \\
& +\mathrm{x}_{1} \mathrm{x}_{4}+\mathrm{x}_{2} \mathrm{x}_{4}+\mathrm{x}_{3} \mathrm{x}_{4}+\mathrm{x}_{4}^{2}+\mathrm{z}_{1}^{2}+\mathrm{z}_{1} \mathrm{z}_{2}+\mathrm{z}_{2}^{2}+\mathrm{z}_{1} \mathrm{z}_{3}+ \\
& \left.+\mathrm{z}_{2} \mathrm{z}_{3}+\mathrm{z}_{3}^{2}+\mathrm{z}_{1} \mathrm{z}_{4}+\mathrm{z}_{2} \mathrm{z}_{4}+\mathrm{z}_{3} \mathrm{z}_{4}+\mathrm{z}_{4}^{2}\right) / 60 \\
& \mathrm{c}=\mu \cdot|\operatorname{DET}(\mathrm{J})| \cdot\left(\mathrm{x}_{1}^{2}+\mathrm{x}_{1} \mathrm{x}_{2}+\mathrm{x}_{2}^{2}+\mathrm{x}_{1} \mathrm{x}_{3}+\mathrm{x}_{2} \mathrm{x}_{3}+\mathrm{x}_{3}^{2}+\mathrm{x}_{1} \mathrm{x}_{4}+\right. \\
& +\mathrm{x}_{2} \mathrm{x}_{4}+\mathrm{x}_{3} \mathrm{x}_{4}+\mathrm{x}_{4}^{2}+\mathrm{y}_{1}^{2}+\mathrm{y}_{1} \mathrm{y}_{2}+\mathrm{y}_{2}^{2}+\mathrm{y}_{1} \mathrm{y}_{3}+ \\
& \left.+\mathrm{y}_{2} \mathrm{y}_{3}+\mathrm{y}_{3}^{2}+\mathrm{y}_{1} \mathrm{y}_{4}+\mathrm{y}_{2} \mathrm{y}_{4}+\mathrm{y}_{3} \mathrm{y}_{4}+\mathrm{y}_{4}^{2}\right) / 60 \\
& +2 \mathrm{a}^{\prime}=\mu \cdot|\operatorname{DET}(\mathrm{J})| \cdot\left(2 \mathrm{y}_{1} \mathrm{z}_{1}+\mathrm{y}_{2} \mathrm{z}_{1}+\mathrm{y}_{3} \mathrm{z}_{1}+\mathrm{y}_{4} \mathrm{z}_{1}+\mathrm{y}_{1} \mathrm{z}_{2}+\right. \\
& \left.+\mathrm{y}_{4} \mathrm{z}_{2}+\mathrm{y}_{3} \mathrm{z}_{2}+\mathrm{y}_{4} \mathrm{z}_{2}+\mathrm{y}_{1} \mathrm{z}_{3}+\mathrm{y}_{2} \mathrm{z}_{3}+2 \mathrm{y}_{2} \mathrm{z}_{4}+\mathrm{y}_{3} \mathrm{z}_{4}+2 \mathrm{y}_{4} \mathrm{z}_{4}\right) / 120 \\
& +2 \mathrm{x}_{2} \mathrm{y}_{2}+\mathrm{x}_{3} \mathrm{y}_{2}+\mathrm{x}_{4} \mathrm{y}_{2}+\mathrm{x}_{1} \mathrm{y}_{3}+\mathrm{x}_{2} \mathrm{y}_{3}+2 \mathrm{x}_{3} \mathrm{y}_{3}+ \\
& b^{\prime}=\mu \cdot|\operatorname{DET}(\mathrm{J})| \cdot\left(2 \mathrm{x}_{1} \mathrm{z}_{1}+\mathrm{x}_{2} \mathrm{z}_{1}+\mathrm{x}_{3} \mathrm{z}_{1}+\mathrm{x}_{2} \mathrm{z}_{4}+\mathrm{y}_{1} \mathrm{z}_{2}+\right. \\
& \left.+2 \mathrm{x}_{3} \mathrm{y}_{4}+2 \mathrm{x}_{4} \mathrm{y}_{4}\right) / 120 \\
& c^{\prime}=\mu \cdot|\operatorname{DET}(\mathrm{J})| \cdot\left(2 \mathrm{x}_{1} \mathrm{y}_{1}+\mathrm{x}_{2} \mathrm{y}_{1}+\mathrm{x}_{3} \mathrm{z}_{2}+\mathrm{x}_{4} \mathrm{z}_{2}+\mathrm{x}_{1} \mathrm{z}_{3}+\mathrm{x}_{2} \mathrm{z}_{3}+2 \mathrm{x}_{3} \mathrm{z}_{3}+\right.
\end{aligned}
$$

Equations (9) give the components of the inertia tensor as quadratic polynomials of the vertex coordinates multiplied by six times the tetrahedron volume and by the density of the medium.

Numerical Example: Consider a tetrahedron with vertex coordinates: 
$A_{1} \equiv\left(\tilde{x}_{1}, \tilde{y}_{1}, \tilde{z}_{1}\right)=(8.33220,-11.86875,0.93355) \mathrm{m} ;$

$A_{2} \equiv\left(\tilde{x}_{2}, \tilde{y}_{2}, \tilde{z}_{2}\right)=(0.75523,5.00000,16.37072) \mathrm{m}$;

$A_{3} \equiv\left(\tilde{x}_{3}, \tilde{y}_{3}, \tilde{z}_{3}\right)=(52.61236,5.00000,-5.38580) \mathrm{m}$;

$A_{4} \equiv\left(\tilde{x}_{4}, \tilde{y}_{4}, \tilde{z}_{4}\right)=(2.00000,5.00000,3.00000) \mathrm{m}$. The

coordinates of the centroid can be calculated as [12]:

$G \equiv\left(\tilde{x}_{G}, \tilde{y}_{G}, \tilde{z}_{G}\right)=(15.92492,0.78281,3.72962) \mathrm{m}$. The

values of the components of the inertia tensor calculated with respect to the centroid, $\mathbf{E}_{\mathrm{G}}$, result as follows: $\mathrm{a} / \mu=$ $43520.33257 \mathrm{~m}^{5} ; \quad \mathrm{b} / \mu=194711.28938 \mathrm{~m}^{5} ; \mathrm{c} / \mu=$ $191168.76173 \mathrm{~m}^{5} ; \mathrm{a}^{\prime} / \mu=4417.66150 \mathrm{~m}^{5} ; \mathrm{b}^{\prime} / \mu=-$ $46343.16662 \mathrm{~m}^{5} ; \mathrm{c}^{\prime} / \mu=11996.20119 \mathrm{~m}^{5}$.

\section{REFERENCES}

1. Arnold, V.I., 1989. Mathematical Methods of Classical Mechanics. Springer, New York.

2. Coxeter, H.S.M., 1973. Regular Polytopes. 3rd Edn. Dover, New York.

3. Hatzor, Y. and R.E. Goodman, 1993. Determination of the "design block" for tunnel supports in highly jointed rock. In Comprehensive Rock Engineering, 2: 263-292.

4. Tonon, F., 1998. Generalization of Mauldon's and Goodman's vector analysis of keyblock rotations. ASCE J. Geotech. Geoenviron. Engg., 124: 913-922.

5. Bern, M., 1997. Triangulation, In Handbook of Discrete and Computational Geometry, (Edited by J.E. Goodman and J. O'Rourke), pp: 413428.
6. Rathod, H.T. and H.S. Govinda Rao, 1995. Integration of polynomials over linear polyhedra in Euclidean three-dimensional space, Computer Methods in Applied Mechanics and Engineering, 126: 373-392.

7. Rathod, H.T. and H.S. Govinda Rao, 1996. Integration of polynomials over an arbitrary tetrahedron in Euclidean three-dimensional space. Comput. \& Structures 59: 55-65.

8. Rathod, H.T. and S.V. Hiremathb, 1998. Boundary integration of polynomials over an arbitrary linear tetrahedron in Euclidean threedimensional space, Computer Methods in Applied Mechanics and Engineering, 153: 81106.

9. Rathod, H. T. and S.V. Hiremathb, 1998. Boundary integration of polynomials over an arbitrary linear hexahedron in euclidean threedimensional space. Computer Methods in Applied Mechanics and Engineering, 161:155193.

10. G. Grioli, Lezioni di Meccanica Razionale, Edizioni Libreria Cortina, Padua, Italy (1988).

11. A. Chiffi, Analisi matematica, Vol. 2, Editrice Alceo, Padua, Italy (1983).

12. Mammino, A. and F. Tonon, 1997. Opere Strutturali per l'Ingegneria Territoriale, Vol.1, Tomo 2 - Le pere in Sotterraneo in Roccia: Alcuni Metodi di Progetto e di Verifica, Alinea, Florence, Italy. 